\title{
Assessing Tuberculosis among Smokers: Secondary Analysis of an Active Case Finding Project
}

\author{
Razia Fatima1, Aashifa Yaqoob ${ }^{1 *}$, Kamran Siddiqi², Omara Dogar' ${ }^{2}$, Sarwat Shah' ${ }^{2}$, Aamer Ikram ${ }^{1,3}$ \\ ${ }^{1}$ Common Management Unit (HIV/AIDs, TB \& Malaria), Islamabad, Pakistan \\ ${ }^{2}$ University of York, Heslington, UK \\ ${ }^{3}$ National Institute of Health, Islamabad, Pakistan \\ Email: ^aashifa.yaqoob@gmail.com
}

How to cite this paper: Fatima, R., Yaqoob, A., Siddiqi, K., Dogar, O., Shah, S. and Ikram, A. (2019) Assessing Tuberculosis among Smokers: Secondary Analysis of an Active Case Finding Project. Journal of Tuberculosis Research, 7, 246-252. https://doi.org/10.4236/jtr.2019.74024

Received: July 11, 2019

Accepted: December 20, 2019

Published: December 23, 2019

Copyright $\odot 2019$ by author(s) and Scientific Research Publishing Inc. This work is licensed under the Creative Commons Attribution International License (CC BY 4.0).

http://creativecommons.org/licenses/by/4.0/

\begin{abstract}
Background: Previous case-control studies and a small number of cohort studies in high-risk populations have found an association between smoking and tuberculosis, but limited studies are available in the general population that predicts association of smoking and TB. Objectives: To investigate the association between smoking and tuberculosis in a cohort of a general population. Methods: Four districts of Punjab province (Lahore, Rawalpindi, Faisalabad and Islamabad) were randomly selected. In routine, household contact investigation is practiced by the National TB Control Programme. For two years from July 2013-June 2015, all people who were living within 50 metres from the household of smear positive TB patients were screened for tuberculosis. Those found presumptive TB were investigated through smear microscopy and those found smear negative; the Xpert MTB/RIF test was done. All the diagnosed TB patients were referred to nearest basic health unit for further treatment and care. Results: A total of 783,043 contacts were screened for tuberculosis, of whom 19,815 (2.53\%) were smokers. Smoking was common among men, in diabetic \& teenage, elderly age population and in household contact of smear positive TB patients. Smoking was associated with an increased risk of tuberculosis (odds ratio [OR], 2.43; 95\% confidence interval, 2.27 - 2.60) in household contact of smear positive TB patients. The association was stronger among those greater than 45 years of age (OR, 11.09) than those between 25 - 44 years of age (OR, 5.83) and diabetic persons (OR, 2.0). Conclusions: Smoking was associated with a twofold increased risk of active tuberculosis in a cohort of general population.
\end{abstract}

\section{Keywords}

Smoking, Tuberculosis, Close Contacts, Pakistan 


\section{Introduction}

According to World Health Organization (WHO), tuberculosis (TB) is among the top ten causes of mortality worldwide and ranks second after cardiovascular disease in most of high-burden countries [1]. Despite the improvement in case detection and treatment, the global incidence of TB remains stagnant over last decade. Preventive measures that address determinants of TB in addition to curative interventions could be more effective in order to reach the goal of END TB strategy by 2035 as compared to curative interventions. An important step of END TB strategy is to better understand the effects of modifiable risk factors and comorbidities on $\mathrm{TB}$, including smoking that could ultimately increase the occurrence of TB. TB patients who smoke have an increased risk of treatment failure, delayed sputum conversion, relapse, and mortality [2] [3] [4]. Major potential confounders include sex, age, residing in a crowded home, income level, marital status, education level, use of alcohol and employment status [5] [6].

According to WHO, over 1.1 billion people smoked tobacco in 2015 and nearly $80 \%$ of them live in low- or middle-income countries [7]. TB and tobacco is responsible for almost over $20 \%$ of TB-related deaths, which could exceed to 40 million TB deaths by 2050 [8]. Several systematic reviews and meta-analysis of observational studies suggested a positive association of smoking with TB acquisition and negative treatment outcomes that may affect rates of TB morbidity and mortality [9] [10] [11] [12]. However, evidence of impact of smoking on TB transmission is insufficient with limitation of retrospective case-control or cross-sectional study design, and cohort studies are limited to high-risk population that are not generalizable, and their results might be biased. There is dearth of studies on general population with large sample size. In light of existing evidence and knowledge gap, the current study is based on a long term follow-up of TB patients and investigation of their household and community living within 50 meter radius of these patients that are representative of the general population in urban areas of Punjab, Pakistan. The study aims to explore the association between smoking and TB in general population living in slum areas of $\mathrm{Pa}$ kistan.

\section{Methods}

This study involved a retrospective record review of a cohort of community contacts living within the 50-meter radius of index TB cases through intensified case finding project [13]. This study was done in slums from July 2013-June 2015 and four districts (Lahore, Rawalpindi, Faisalabad and Islamabad) were randomly selected from province Punjab, Pakistan. In which contact investigation beyond household was conducted and all people staying within a radius of 50 meters from the household of smear positive TB patients were screened for tuberculosis using a short symptom-screening questionnaire. The questionnaire (using mobile phones enabled with geographic information system (GIS) technology) assessed current TB treatment status, presence of cough more than two 
weeks, blood streak sputum, fever, unexplained weight loss, contact with a TB patient, self-reported smoking status (cigarette smoker? Yes/No) and diabetes status. Smokers were defined as participants who reported that they were current cigarette smokers. Algorithm of the project is given in Figure 1. Any person with productive cough for more than two weeks was defined as "presumptive TB patient". Those with presumptive TB were investigated using smear microscopy and the Xpert MTB/RIF test was performed on smear negative patients. All the diagnosed TB patients were linked to TB treatment and care. Child presumptive TB patients were referred to nearest basic medical unit for investigations.

The analysis was done in STATA version 12 (StataCorp., College Station, TX, USA). The presence of an association between smoking and TB was explored using multivariate logistic regression model, using self-reported smoking status and other covariate as the exposures to determine the independent predictors of TB \& smoking, assess for confounding and statistical interaction. P-value < 0.005 was considered as significance.

\section{Result}

A total of 783,043 contacts were screened for tuberculosis symptoms, of whom 19,815 (2.53\%) were smokers. Table 1 shows the characteristics of smokers and non-smokers. There were significant variation in characteristics of smokers and non-smokers when disaggregated by sex, age, diabetic status and TB contact. Cigarette smoking consumption were common among men, in diabetic \& teenage, elderly age population and in household contact of index TB patients. Table 2 shows the independent association of smoking status and other covariates with TB. Smoking diabetes, being male and being household contact of Index TB case are independently associated with TB. Smoking was associated with an increased risk of tuberculosis (odds ratio [OR], 2.43; 95\% confidence interval, 2.27 - 2.60) in household contact of smear positive TB patients. The association was stronger among those greater than 45 years of age $(\mathrm{OR}, 11.09)$ than those between 25 - 44 years of age (OR, 5.83) and diabetic persons (OR, 2.0).

\section{Discussion}

The prevalence of smokers in adult general population in Pakistan is 19.1\% [14]. In this study, we found a threefold increase in the risk of TB in current smokers compared with never smokers, which was significantly less than the previous study. A review of the literature suggests a positive association between current smoking and TB [5] [11] [15]. The observed magnitude of association ranged from 1.73 to 2.68 . The results from our study are consistent with the positive association found in other studies. Our study reveals that the association between smoking and TB might differ by age, with a higher OR for those greater than 45 years of age. Other studies on the associations between smoking and TB and other diseases e.g. diabetic [16], have reported decreasing relative risks by age. The differential association by age observed in our study that smoking may be a 
stronger risk factor in the elderly results from reactivation of remote infection. Whereas, in contrast to our study finding, in a cohort study in Hong Kong, smokers had a nearly threefold increased risk of TB, but among the elderly OR was 0.78 [17].

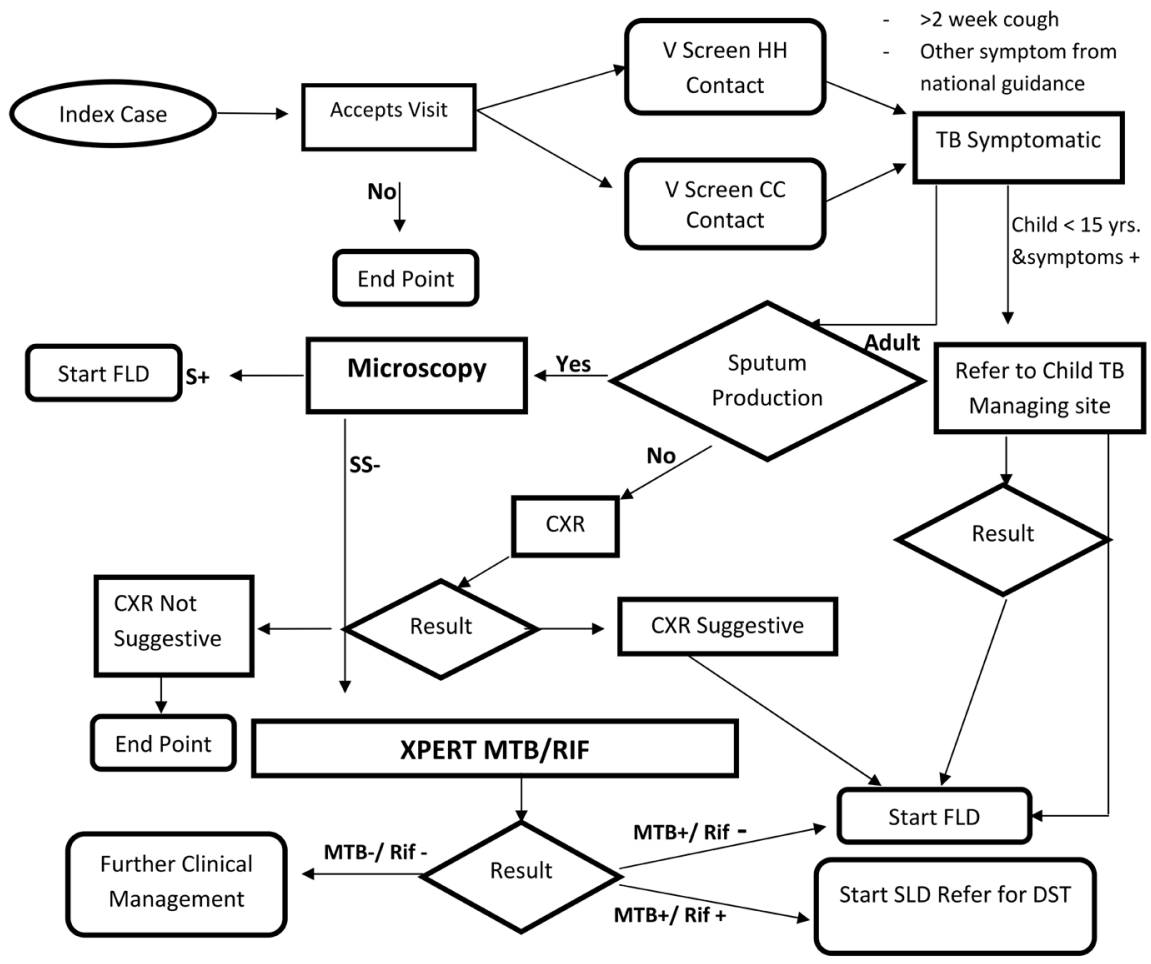

Figure 1. Flow chart to explain the flow of patients [11]. V screen: Verbal screening, HH: Household, CC: Community contact, SS +: sputum smear positive, SS -: Sputum smear negative, CXR: chest X-ray, MTB +: Mycobacterium tuberculosis detected, MTB -: Mycobacterium tuberculosis not detected, Rif + : resistance to rifampin, Rif -: No resistance to rifampin, FLD: First line drug, SLD: Second line drug, DST: Drug susceptible test.

Table 1. Baseline characteristics of active smokers and not smokers.

\begin{tabular}{llll}
\hline Characteristics & Active Smokers & Non Smokers & P-Value \\
\hline Age $(\mathrm{yrs})$ & & & \\
$\bullet \leq 14$ & $649(0.24)$ & $272,958(99.76)$ & \\
$\bullet 15-24$ & $1500(0.84)$ & $176,158(99.16)$ & $0.001<$ \\
$\bullet 25-44$ & $8742(4.09)$ & $204,830(95.91)$ & \\
$\bullet \geq 45$ & $8862(7.57)$ & $108,136(92.43)$ & \\
Gender & & & \\
- Male & $18,026(4.19)$ & $411,830(95.81)$ & $0.001<$ \\
- Female & $1789(0.51)$ & $351,228(99.49)$ & \\
Diabetic & & & \\
- Yes & $2096(28.99)$ & $5135(71.01)$ & $0.001<$ \\
- No & $17,719(2.28)$ & $757,921(97.72)$ & \\
TB Contacts & & & \\
- Household & $3014(3.38)$ & $86,180(96.62)$ & $0.001<$ \\
- Community & $16,801(2.42)$ & $676,878(97.58)$ & \\
Total & $19,815(2.53)$ & $763,058(97.47)$ & \\
\hline
\end{tabular}


Table 2. Association of smoking status and other covariates with tuberculosis.

\begin{tabular}{lll}
\hline Characteristics & Odds Ratio & Confidence Interval \\
\hline Age & & \\
$\bullet \leq 14$ (Reference) & & \\
$\bullet 15-24$ & 6.81 & $(5.9-7.7)$ \\
$\bullet 25-44$ & 5.83 & $(5.1-6.6)$ \\
$\bullet \geq 45$ & 11.09 & $(9.8-12.6)$ \\
Gender (Female as reference) & & \\
$\bullet$ Male & 1.10 & $1.04-1.17$ \\
Smokers & 2.72 & $2.4-3.0$ \\
Diabetic & 2.01 & $1.7-2.3$ \\
TB Contacts (community as reference) & & \\
$\bullet$ & 2.43 & $2.27-2.60$ \\
\hline
\end{tabular}

All the covariates were controlled for in the same model.

The prevalence and notification rates of TB in Pakistan are higher among men [18]. In our study population, proportion of smokers among men was substantially higher than women. This suggests that smoking may be the underlying cause of the sex difference in TB. Whereas, the study results shows that index household and smoking are the main risk factors for developing TB. It is advisable to ask smoking status at initial screening phase of index household then close community phase in routine setting as a part of national strategy so that reliable estimates of smoking prevalence among TB patients can be available at national level.

A major strength of this study is the follow up of large scale general population and unlike previous cohort, studies that were only targeted the high-risk populations, this study was conducted in a general community with larger sample size and has greater generalizability. Moreover, the National TB control program was leading the implementation of project and this is the first study in general population in Pakistan with active case finding technique that provides evidence on the positive association between smoking and TB. The primary objective of the study was not to assess the association of smoking and TB so it is difficult to ensure the accuracy and completeness of data related to smoking. Moreover, only history of TB was taken, duration and intake of smoking were not recorded. Based on our study finding, it is recommended to record smoking status at initial screening phase in routine practice and an integrated approach can be proposed for smoking cessation in TB care as part of TB control [19]. This analysis is never done as part of NTP project to assess the association TB and smoking. The findings will help the program to invest on designing cost effective intervention and behavior support for TB patients to quit smoking while initiating TB treatment. Further large scale research studies/survey are proposed to determine the exact prevalence of smoking among TB patients.

\section{Conclusions}

Smoking was associated with a two-fold increased risk of active tuberculosis and 
there is need for targeted smoking cessation strategies for elderly smokers and diabetic among TB patients.

Behavior support for TB patients to quit smoking should be encouraged in routine settings by National TB Control Program.

\section{Acknowledgements}

This research was conducted through the Structured Operational Research and Training Initiative (SORT IT), a global partnership led by the Special Programme for Research and Training in Tropical Diseases at the World Health Organization (WHO/TDR). The SORT-IT course is developed by the International Union against Tuberculosis and Lung Disease (The Union, Paris, France) and Médecins Sans Frontières (MSF, Geneva, Switzerland). The specific SORT IT programme that resulted in this publication was implemented by the National Tuberculosis Control Programme of Pakistan, through the support of the Global Fund to Fight AIDS, Tuberculosis and Malaria (The Global Fund, Geneva, Switzerland). The publication fee was covered by the Special Programme for Research and Training in Tropical Diseases at the World Health Organization (WHO/TDR).

\section{Conflicts of Interest}

The authors declare no conflicts of interest regarding the publication of this paper.

\section{References}

[1] WHO (2009) Global Health Risks: Mortality and Burden of Disease Attributable to Selected Major Risks. Bulletin of the World Health Organization, 87, 646-646. https://doi.org/10.2471/BLT.09.070565

[2] Batista, J.D.A.L., Militão De Albuquerque, M.F.P., De Alencar Ximenes, R.A. and Rodrigues, L.C. (2008) Smoking Increases the Risk of Relapse after Successful Tuberculosis Treatment. International Journal of Epidemiology, 37, 841-851. https://doi.org/10.1093/ije/dyn113

[3] Santha, T., Garg, R., Frieden, T.R., Chandrasekaran, V., Subramani, R., Gopi, P.G., et al. (2002) Risk Factors Associated with Default, Failure and Death among Tuberculosis Patients Treated in a DOTS Programme in Tiruvallur District, South India, 2000. International Journal of Tuberculosis and Lung Disease, 6, 780-788.

[4] Güler, M., Unsal, E., Dursun, B., Aydln, O. and Capan, N. (2007) Factors Influencing Sputum Smear and Culture Conversion Time among Patients with New Case Pulmonary Tuberculosis. International Journal of Clinical Practice, 61, 231-235. https://doi.org/10.1111/j.1742-1241.2006.01131.x

[5] Lin, H.H., Ezzati, M., Chang, H.Y. and Murray, M. (2009) Association between Tobacco Smoking and Active Tuberculosis in Taiwan: Prospective Cohort Study. American Journal of Respiratory and Critical Care Medicine, 180, 475-480. https://doi.org/10.1164/rccm.200904-0549OC

[6] Soh, A.Z., Chee, C.B.E., Wang, Y.T., Yuan, J.M. and Koh, W.P. (2017) Alcohol Drinking and Cigarette Smoking in Relation to Risk of Active Tuberculosis: Prospective Cohort Study. BMJ Open Respiratory Research, 4, e000247. 
https://doi.org/10.1136/bmjresp-2017-000247

[7] World Health Organization (2017) Fact Sheet: Tobbaco [Internet]. WHO, Geneva.

[8] Basu, S., Stuckler, D., Bitton, A. and Glantz, S.A. (2011) Projected Effects of Tobacco Smoking on Worldwide Tuberculosis Control: Mathematical Modelling Analysis. BMJ, 343, d5506-d5506. https://doi.org/10.1136/bmj.d5506

[9] Lin, H.-H., Ezzati, M. and Murray, M. (2007) Tobacco Smoke, Indoor Air Pollution and Tuberculosis: A Systematic Review and Meta-Analysis. PLOS Medicine, 4, e20. https://doi.org/10.1371/journal.pmed.0040020

[10] Slama, K., Chiang, C.-Y., Enarson, D., Hassmiller, K., Fanning, A., Gupta, P., et al. (2007) Tobacco and Tuberculosis: A Qualitative Systematic Review and Meta-Analysis. International Journal of Tuberculosis and Lung Disease, 11, 1049-1061.

[11] Bates, M.N., Khalakdina, A., Pai, M., Chang, L., Lessa, F. and Smith, K.R. (2007) Risk of Tuberculosis from Exposure to Tobacco Smoke: A Systematic Review and Meta-Analysis. Archives of Internal Medicine, 167, 335-342. https://doi.org/10.1001/archinte.167.4.335

[12] Alavi-Naini, R., Moghtaderi, A., Metanat, M., Mohammadi, M. and Zabetian, M. (2013) Factors Associated with Mortality in Tuberculosis Patients. Journal of Research in Medical Sciences, 18, 52-55.

[13] Fatima, R., Qadeer, E., Yaqoob, A., Ul Haq, M., Majumdar, S.S., Shewade, H.D., et al. (2016) Extending "Contact Tracing" into the Community within a 50-Metre Radius of an Index Tuberculosis Patient Using Xpert MTB/RIF in Urban, Pakistan: Did It Increase Case Detection? PLoS ONE, 11, e0165813. https://doi.org/10.1371/journal.pone.0165813

[14] Pakistan Health Research Council (2014) Global Adult Tobacco Survey Pakistan.

[15] Petersen, A.B., Muffley, N., Somsamouth, K. and Singh, P.N. (2019) Smoked Tobacco, Air Pollution, and Tuberculosis in Lao PDR: Findings from a National Sample. International Journal of Environmental Research and Public Health, 16, 3059.

[16] Jeon, C.Y. and Murray, M.B. (2008) Diabetes Mellitus Increases the Risk of Active Tuberculosis: A Systematic Review of 13 Observational Studies. PLOS Medicine, 5, 1091-1101. https://doi.org/10.1371/journal.pmed.0050181

[17] Leung, C.C., Li, T., Lam, T.H., Yew, W.W., Law, W.S., Tam, C.M., et al. (2004) Smoking and Tuberculosis among the Elderly in Hong Kong. American Journal of Respiratory and Critical Care Medicine, 170, 1027-1033. https://doi.org/10.1164/rccm.200404-512OC

[18] Qadeer, E., Fatima, R., Yaqoob, A., Tahseen, S., Ul Haq, M., Ghafoor, A., et al. (2016) Population Based National Tuberculosis Prevalence Survey among Adults (>15 Years) in Pakistan, 2010-2011. PLoS ONE, 11, e0148293. https://doi.org/10.1371/journal.pone.0148293

[19] WHO (2014) A Guide for Tuberculosis Patients to Quit Smoking. 